\title{
Probabilistic Registration of an Unbiased Statistical Shape Model to Ultrasound Images of the Spine
}

\author{
Abtin Rasoulian $^{a}$, Robert N. Rohling ${ }^{a, b}$, and Purang Abolmaesumi ${ }^{a}$ \\ ${ }^{a}$, Department of Electrical and Computer Engineering, University of British Columbia, \\ Vancouver, BC, CANADA \\ ${ }^{b}$ Department of Mechanical Engineering, University of British Columbia, Vancouver, BC, \\ CANADA
}

\begin{abstract}
The placement of an epidural needle is among the most difficult regional anesthetic techniques. Ultrasound has been proposed to improve success of placement. However, it has not become the standard-of-care because of limitations in the depictions and interpretation of the key anatomical features. We propose to augment the ultrasound images with a registered statistical shape model of the spine to aid interpretation. The model is created with a novel deformable group-wise registration method which utilizes a probabilistic approach to register groups of point sets. The method is compared to a volume-based model building technique and it demonstrates better generalization and compactness. We instantiate and register the shape model to a spine surface probability map extracted from the ultrasound images. Validation is performed on human subjects. The achieved registration accuracy $(2-4 \mathrm{~mm})$ is sufficient to guide the choice of puncture site and trajectory of an epidural needle.
\end{abstract}

Keywords: Statistical Shape Model, 3D Ultrasound, Spine, Registration

\section{INTRODUCTION}

Epidural anesthesia is a form of regional anesthesia involving placement of a needle into the epidural space (Figure 1). Placement of an epidural is among the most difficult regional anesthetic techniques due to the narrow intervertebral foramen. Currently, experience on at least 60 subjects is required to reach competency of $90 \%$ in epidural needle placement. ${ }^{1}$ Ultrasound has been proposed for pre-puncture guidance, ${ }^{2}$ but it has not become standard-of-care because of limitation in image interpretation by anesthesiologists unfamiliar with complex spinal sonography. The challenge is to identify the echo from the target ligamentum flavum/epidural space interface, among a complex set of echoes. To improve guidance with ultrasound imaging, augmenting ultrasound images with registered pre-operative data such as CT has been suggested. ${ }^{3}$ However, pre-operative images are not normally available, especially in obstetrics. Hence, the use of statistical shape models (SSMs, or atlases) is a reasonable alternative. The accuracy of the SSM and its registration to ultrasound needs to be sufficient for correct identification of the needle entry point and trajectory. During needle insertion, real-time ultrasound will be combined with traditional loss-of-resistance technique to confirm correct needle insertion depth, and to avoid overshoot of the needle into the dura mater, which can result in nerve injury to the spinal cord. ${ }^{2}$ Given this paradigm, we selected the acceptable accuracy for the registered SSM to be $4 \mathrm{~mm}$, which is less than half of the distance between ligamentum flavum and the dura mater. ${ }^{4}$ This threshold is also helpful to reliably distinguish the US echo of the target ligamentum flavum, located on the posterior side of vertebrae, from nearby echoes. An SSM usually includes a mean shape and its principal variations; capable of generating an unseen instance of a specific anatomy. Many methods have been proposed to reconstruct SSMs of bony anatomies. ${ }^{5,6}$ These approaches generally use a template-based algorithm to construct the SSM where all the training examples are registered to an image template to create the correspondences of anatomical points. This creates a bias in the SSM towards the shape of the template itself, which may introduce larger registration errors when the unseen observation (i.e. the patient's anatomy) is far from the template. ${ }^{7}$ To avoid this problem, group-wise registration of all training data to each other and simultaneously constructing the mean shape is suggested in other contexts. Several group-wise SSM generation techniques based on volumetric registration, ${ }^{7,8}$ or surface registration, ${ }^{9}$ of training set have been previously proposed.

\footnotetext{
Medical Imaging 2012: Image-Guided Procedures, Robotic Interventions, and Modeling, edited by David R. Holmes III, Kenneth H. Wong, Proc. of SPIE Vol. 8316, 83161P (c) 2012 SPIE · CCC code: $1605-7422 / 12 / \$ 18 \cdot$ doi: $10.1117 / 12.911742$
} 


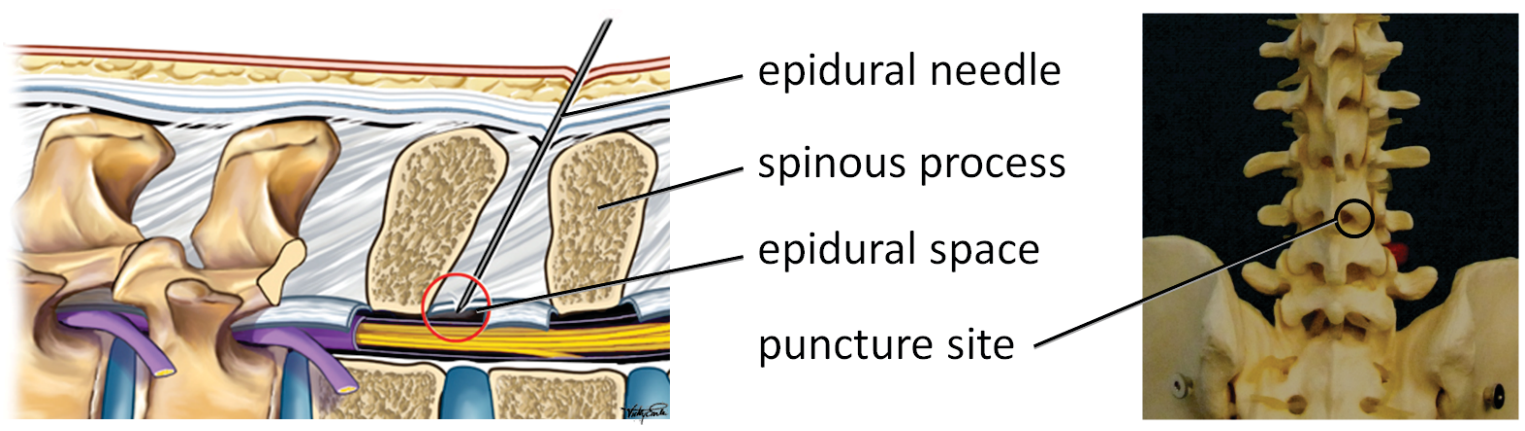

Figure 1: Sagittal view of the vertebra column and epidural needle injection.

Previous attempts for registering SSMs to ultrasound data ${ }^{5,6}$ suggest that, without hardware acceleration, registering volumetric atlases to ultrasound images is computationally more expensive than registering atlases based on surface data. In search for a clinically viable solution for real-time registration of an unbiased SSM to ultrasound data, we propose a method to create an unbiased SSM using a novel surface-based group-wise registration method. This technique extends the affine group-wise registration method proposed by Hufnagel et al. ${ }^{9}$ to a deformable method by integrating the deformable registration technique proposed by Myronenko et al. ${ }^{10}$ We also develop a probabilistic technique to register the SSM to ultrasound images. Our approach aims to be substantially faster than the one proposed by Khallaghi et al., ${ }^{6}$ which is a volumetric atlas-based registration.

\section{METHODS}

\subsection{SSM Construction}

An affine group-wise algorithm, ${ }^{9}$ which utilizes a probabilistic approach to register group of point sets, is extended to a deformable method and is used to generate the shape model of the vertebra. Each training data is a point set presented in $D$ dimensions. Assume that there are $K$ point sets in the training set, where $\mathbf{T}_{k}$ is the $k^{t h}$ training point set, presented with $N_{k}$ points, $t_{n}^{k}$. The mean shape, $\mathbf{Z}$, is presented with $M$ points, $z_{m}$ ( $M$ and $N_{k}$ are not necessarily equal). The problem is formulated as a probability density estimation problem, where the mean shape represents the Gaussian Mixture Model (GMM) centroid, and each training point set represents a data point generated by this model. The mean shape, $\mathbf{Z}$, and its transformations to instances, $\Phi_{k}$, can be found by minimizing the negative log-liklihood function:

$$
E\left(\Phi_{k}, \mathbf{Z}\right)=-\sum_{k=1}^{K} \sum_{n=1}^{N_{k}} \log \sum_{m=1}^{M} P(m) p\left(t_{n}^{k} \mid \Phi_{k}\left(z_{m}\right)\right),
$$

This is minimized using an Expectation-Maximization algorithm. ${ }^{10}$ The expectation part computes the probability of how likely a point in the mean shape generates another point in a training point set:

$$
\left.P^{(} z_{m} \mid t_{n}^{k}\right)=\frac{\exp \left(-\frac{1}{2}\left\|\frac{t_{n}^{k}-\Phi_{k}\left(z_{m}\right)}{\sigma}\right\|^{2}\right)}{\sum_{j=1}^{N_{k}} \exp \left(-\frac{1}{2}\left\|\frac{t_{j}^{k}-\Phi_{k}\left(z_{m}\right)}{\sigma}\right\|^{2}\right)}
$$

The value of $\sigma$ controls the uncertainty. Initially, $\sigma$ is chosen to be large. Its value decreases in each iteration. Parameters are updated in the Maximization step to minimize the following objective function:

$$
\left(\mathbf{Z}^{*}, \Phi_{k}^{*}\right)=\underset{\mathbf{Z}, \Phi_{k}}{\operatorname{argmin}} \sum_{k=1}^{K} \frac{1}{2 \sigma_{k}^{2}} \sum_{m, n=1}^{M, N_{k}} P\left(m \mid t_{n}^{k}\right)\left\|t_{n}^{k}-\Phi_{k}\left(z_{m}\right)\right\|^{2}+\frac{\lambda}{2}\left\|\mathbf{L} \Phi_{k}\right\|^{2} .
$$

The latter term is a regularization over the transformations. The objective function can be minimized alternatively with respect to the mean shape and the transformation parameters. For a fixed mean shape, the problem becomes a registration problem and each term of (3) can be minimized separately. For a given $\mathbf{T}_{k}$ and $\mathbf{Z}$ this 
minimization is exactly a registration problem, as stated and solved by Myronenko et al. ${ }^{10}$ They borrowed the idea of coherent point drift (CPD) to model the deformations and solved the registration step in a closed-form solution. ${ }^{10}$ For fixed transformations, each point $\left(z_{m}\right)$ of the mean shape can be found separately by minimizing:

$$
\sum_{k=1}^{K} \sum_{n=1}^{N_{k}} P\left(m \mid t_{n}^{k}\right)\left\|t_{n}^{k}-\Phi_{k}\left(z_{m}\right)\right\|^{2}
$$

This cost function is minimized by finding $z_{m}$ using the BFGS Quasi-Newton method, with a cubic-line search.

\subsection{Instantiation and registration of the SSM to ultrasound images}

In this work, US volumes are captured by a 3D transducer (4DC7-4/40, Ultrasonix, Rigchmond). We closely follow the approach of Foroughi et al. ${ }^{11}$ to compute the bone surface probability in US images for each pixel by weighted summation of reflection amount (pixel intensity) and shadowing effect. The shadow below a pixel is quantified by a weighted summation of the intensity values of all pixels beneath. The result, $V(X)$, represents the bone surface probability for each pixel in the US volume. Since the US probe is positioned posterior-anterior, only the posterior aspect of the vertebra is visible. Similar to the work of Winter et al. ${ }^{12}$ the points from the anterior surface are removed, leaving those points on the tip of spinous process, laminae and facets.

To register the SSM to the bone surface probability, the model is evolved using $A^{\prime}=R(A+b \Phi)+t$. Here, $R$ and $t$ are the rigid transformation parameters, and $A+b \Phi$ is the deformation of the atlas using modes learned in the training step. Assume that $a_{i}$ are the points belonging to $A^{\prime}$. The following objective function is maximized:

$$
\left(R^{*}, t^{*}, b^{*}\right)=\underset{R, t, b}{\operatorname{argmax}} \sum V\left(a_{i}\right)
$$

All parameters are optimized simultaneously using the CMA-ES optimizer. ${ }^{12}$

Experiments are performed on lumbar and thoracic vertebrae. CT images (with $0.70 \mathrm{~mm} \times 0.70 \mathrm{~mm} \times 2.50 \mathrm{~mm}$ spacing) are collected at a local hospital with approval from the research ethics board, and informed consent. Using ITK-Snap, L1 $(n=37)$ AND T12 $(n=18)$ vertebrae are semi-automatically segmented from these images for generation of the SSM.

\section{RESULTS AND DISCUSSION}

3D ultrasound volumes are captured from the L1 and T12 vertebrae of three human subjects. The transducer is positioned in the transverse plane such that the tip of the spinous process is centered in the image. An expert sonographer is asked to select points on the bone surface belonging to the target vertebra. For each patient, the probability map of the bone surfaces is extracted from the ultrasound volumes. The shape model is registered manually to the segmented points. Then, the model is misaligned by applying a rigid transformation including translation and rotation parameters drawn randomly within $\pm 10 \mathrm{~mm}$ and \pm 10 degrees. The shape model is then registered to the probability map of the ultrasound volume using the proposed method. This experiment is repeated 100 times for each vertebra of each patient (overall 600 registrations are performed).

\subsection{SSM accuracy}

Figure 2 illustrates the first three deformation modes of the SSM of the L1 vertebra. Our proposed method (referred as group-wise CPD) is compared to a volume-based method of atlas generation proposed by Balci et $a l .{ }^{8}$ (referred as congealing) in terms of generalization, $G(M)$, and compactness, $C(M),{ }^{13}$ as a function of the number of shape parameters, $M$, used in the reconstruction (Figure $3 \mathrm{a}$ and $3 \mathrm{~b}$ ). The generalization ability of the atlas is measured by generating the SSM using all but one member of the training set and then finding the RMS distance error of registering the atlas to the excluded member. The compactness is the accumulative variance for the first $n$ modes of the atlas. The model is capable of generating an unseen observation with an RMS distance error of $0.85 \mathrm{~mm}$ using the first four modes. $78 \%$ of the variations are achieved by the first four modes. Given that, four modes are used for registration below. The congealing method generates a model with the same generalization ability but with less compactness. 


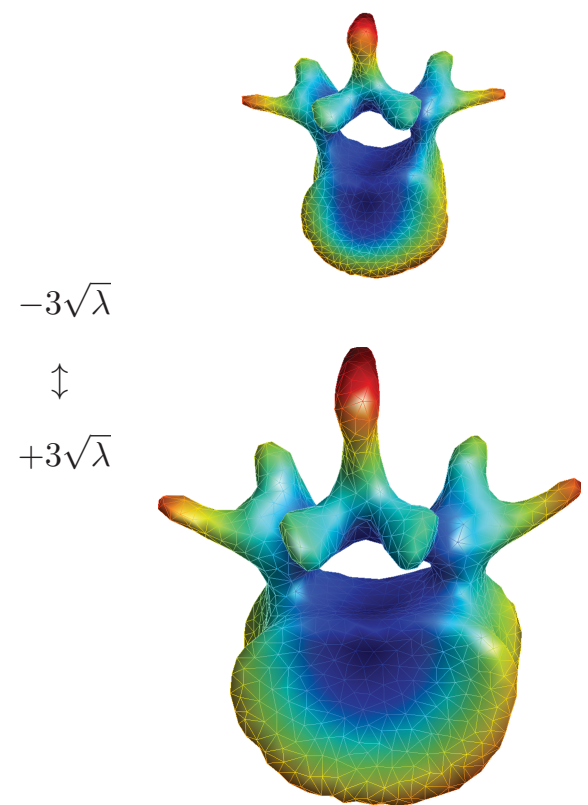

First mode
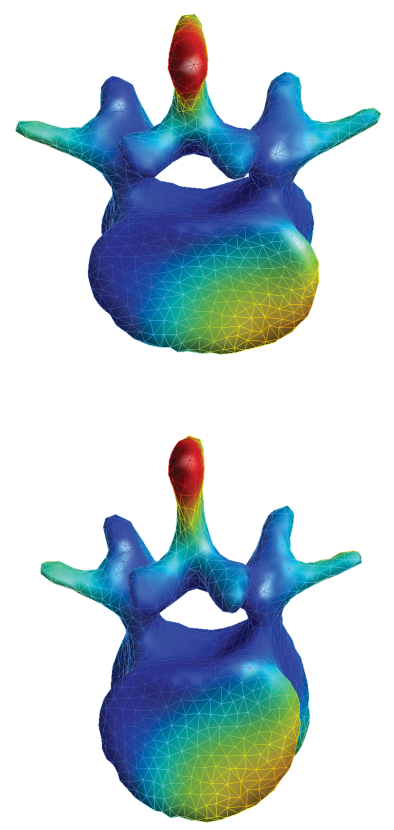

Second mode
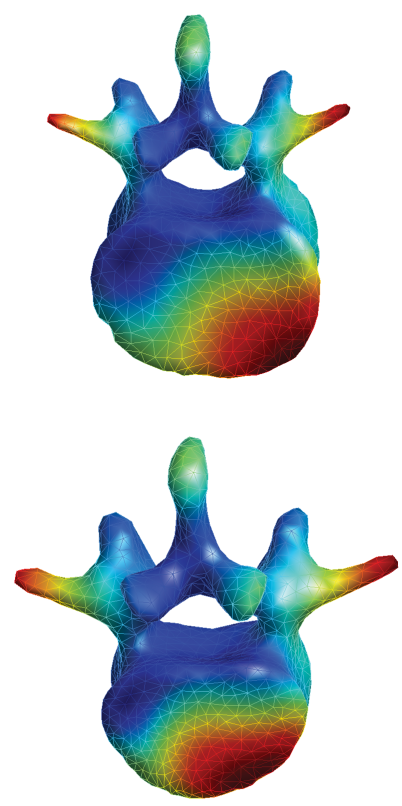

Third mode

Figure 2: Graphical representation of the L2 vertebrae shapes described by the SSM after varying the weights corresponding to the first three and four principal modes of variation by $3 \sigma$. Amount of variation is color coded for each mode. Higher variations are shown in red.

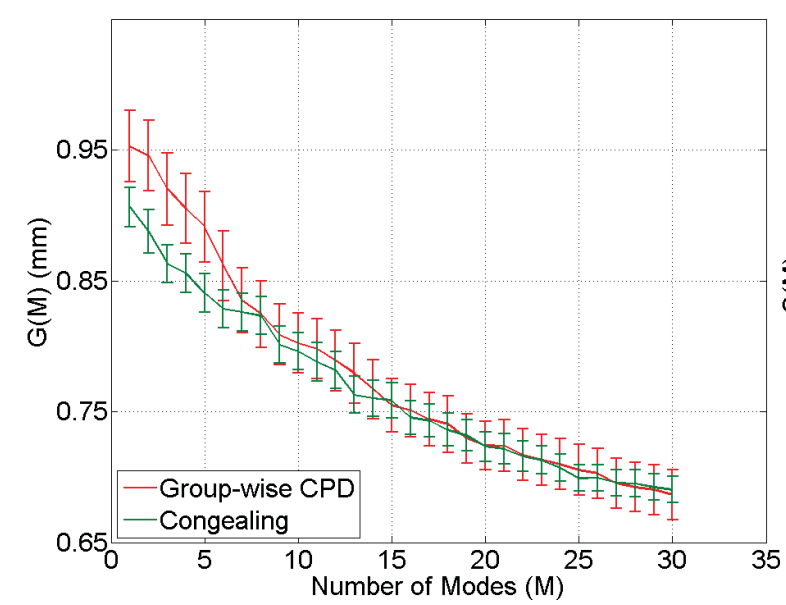

(a) Generalization

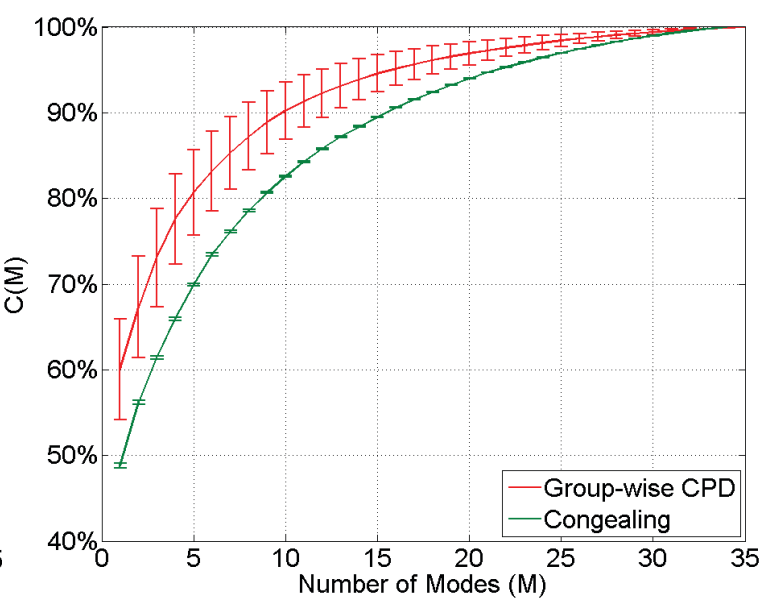

(b) Compactness

Figure 3: a) Generalization and b) Compactness ability of the L1 shape models. Same results are observed for $\mathrm{T} 12$.

\subsection{Registration to in vivo ultrasound images}

Examples of the registration results are shown in Figure 4 . The RMS distance between manually selected points and their nearest point from registered model points is computed as a measure of accuracy in each case. Figure 5 includes results for each patient separately.

Given the overlay of the atlas on the ultrasound, the echoes from the ligamentum flavum/epidural space can be 


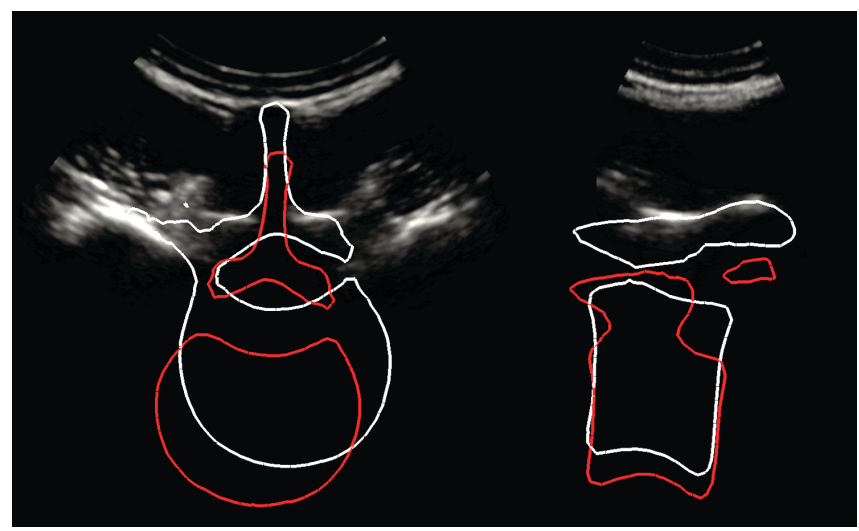

Figure 4: Example of registration of a SSM to ultrasound images of human T12 vertebra. The initial position of the model is drawn in red, the registered model is white, and the CT surface is green.

readily interpreted and localized. It is expected that the SSM will be used to augment the ultrasound image interpretation, but not replace the standard technique for epidural needle placement. The registered SSM, built using the proposed method, had success rate (final average TRE below $4 \mathrm{~mm}$ ) of $84 \%$. The success rate is $74 \%$ for the alternative congealing method. In this pilot study, we have demonstrated that the range of errors for registering the SSM to ultrasound images of human spine is $2.3 \mathrm{~mm}$ to $2.8 \mathrm{~mm}$ for T12 and $3.4 \mathrm{~mm}$ to $4.5 \mathrm{~mm}$ for L1. Our results show that the two group-wise registration methods produce acceptable accuracy for the targeted clinical application. However, the group-wise CPD shows better compromise between generalization and compactness. The current unoptimized MATLAB code requires 183 seconds to register the SSM to 3D ultrasound images compared to many hours in the work by Khallaghi et al. ${ }^{6}$

\section{CONCLUSION AND FUTURE WORK}

A new feature-based method is proposed to construct an unbiased statistical shape model. The method is used to generate the shape model of a single vertebra. The method is compared to the other state-of-the-art technique and shows better compromise between generalization and compactness. A novel technique is proposed to register the model to an in-vivo 3D US volume. The range of errors for registering the SSM to ultrasound images of human spine is $2.3 \mathrm{~mm}$ to $2.8 \mathrm{~mm}$ for T12 and $3.4 \mathrm{~mm}$ to $4.5 \mathrm{~mm}$ for L1, which is close to the required clinical accuracy of $4 \mathrm{~mm}$. Future work will involve optimizing the code on Graphics Processing Unit (GPU) to achieve clinically acceptable runtime.

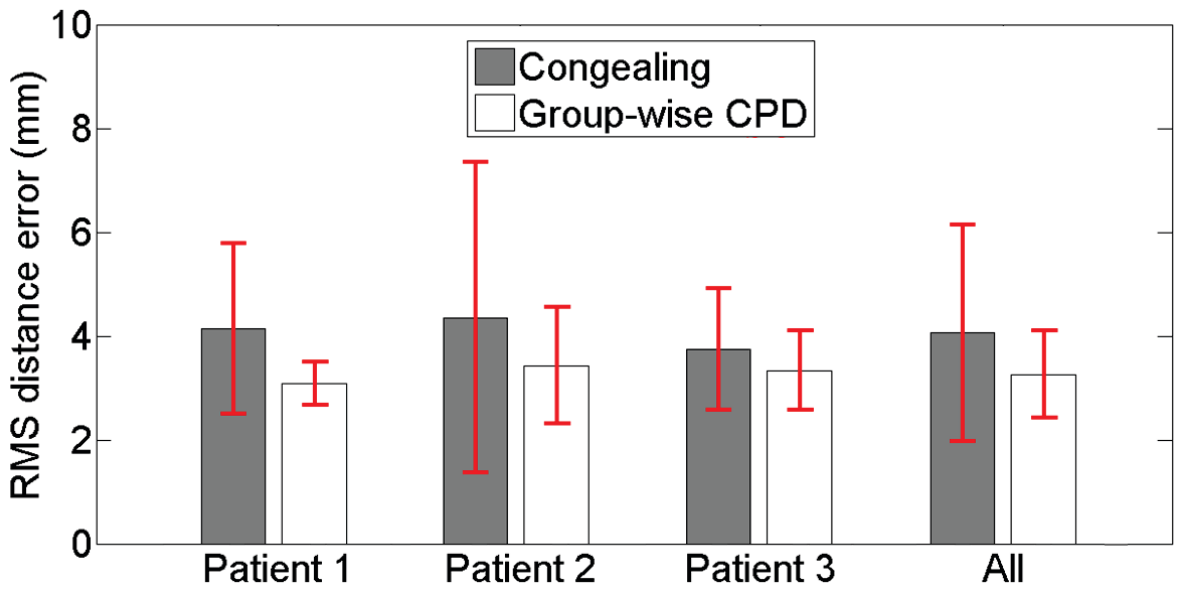

Figure 5: (a) TRE and (b) RMS distance error for probabilistic registration of the model built by our method and the congealing technique to ultrasound images of in vivo data for both L1 and T12 vertebrae. 


\section{STATEMENT OF ORIGINALITY}

The unbiased SSM reconstruction method presented here combined with the probabilistic registration to ultrasound volumes is a novel approach. The SSM is built using a feature-based method and is registered to the bone surface probability map extracted from the ultrasound volume. This work has not previously been submitted for publication

\section{ACKNOWLEDGMENTS}

This study is is jointly funded by grants from the Natural Sciences and Engineering Research Council of Canada (NSERC) and the Canadian Institutes of Health Research (CIHR). The authors would also like to thank sonographer Victoria A. Lessoway for her assistance.

\section{REFERENCES}

[1] Grau, T., Bartusseck, E., Conradi, R., Martin, E., and Motsch, J., "Ultrasound imaging improves learning curves in obstetric epidural anesthesia: a preliminary study," Canadian Journal of Anesthesia 50(10), 1047-1050 (2003).

[2] Tran, D., Kamani, A., Lessoway, V., Peterson, C., Hor, K., and Rohling, R., "Preinsertion paramedian ultrasound guidance for epidural anesthesia," Anesthesia \&3 Analgesia 109(2), 661-667 (2009).

[3] Moore, J., Clarke, C., Bainbridge, D., Wedlake, C., Wiles, A., Pace, D., and Peters, T., "Image guidance for spinal facet injections using tracked ultrasound," in [MICCI], 5761, 516-523 (2009).

[4] Grau, T., Leipold, R., Horter, J., Conradi, R., Martin, E., and Motsch, J., "The lumber epidural space in pregnancy: Visualization by ultrasonography," British Journal of Anesthesia, 798-804 (2001).

[5] Barratt, D., Chan, C., Edwards, P., Penney, G., Slomczykowski, M., Carter, T., and Hawkes, D., "Instantiation and registration of statistical shape models of the femur and pelvis using 3D ultrasound imaging," Medical Image Analysis 12(3), 358 - 374 (2008).

[6] Khallaghi, S., Mousavi, P., Gong, R., Gill, S., Boisvert, J., Fichtinger, G., Pichora, D., Borschneck, D., and Abolmaesumi, P., "Registration of a statistical shape model of the lumbar spine to 3D ultrasound images," 6362, 68-75 (2010).

[7] Bhatia, K., Hajnal, J., Puri, B., Edwards, A., and Rueckert, D., "Consistent groupwise non-rigid registration for atlas construction," IEEE International Symposium on Biomedical Imaging , 908 - 911 (2004).

[8] Balci, S., Golland, P., and III, W. W., "Non-rigid groupwise registration using B-spline deformation model," MICCAI , 105-121 (2007).

[9] Hufnagel, H., Pennec, X., Ehrhardt, J., Ayache, N., and Handels, H., "Generation of a statistical shape model with probabilistic point correspondences and the expectation maximization-iterative closest point algorithm," International Journal of Computer Assisted Radiology and Surgery 2(5), 265-273 (2008).

[10] Myronenko, A., Song, X., and Carreira-Perpinan, M., "Non-rigid point set registration: Coherent point drift," Advances in Neural Information Processing Systems 19, 1009-1016 (2007).

[11] Foroughi, P., Boctor, E., Swartz, M., and et al., "2-D ultrasound bone segmentation using dynamic programming," IEEE Ultrasonics Symposium, 2523-2526 (2007).

[12] Winter, S., Brendel, B., Pechlivanis, I., Schmieder, K., and Igel, C., "Registration of CT and intraoperative 3-D ultrasound images of the spine using evolutionary and gradient-based methods," Evolutionary Computation, IEEE Transactions on 12(3), 284-296 (2008).

[13] Styner, M., Rajamani, K., Nolte, L., Zsemlye, G., Székely, G., Taylor, C., and Davies, R., "Evaluation of 3D correspondence methods for model building," Information Processing in Medical Imaging 2732, 63-75 (2003). 